\title{
LEGIBILITY
}

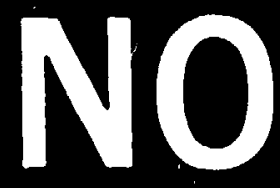

A major purpose of the Technical Information Center is to provide the broadest dissemination possible of information contained in DOE's Research and Development Reports to business, industry, the academic community, and federal, state and local governments.

Although a small portion of this report is not reproducible, it is being made available to expedite the availability of information on the research discussed herein. 
LA-UA $-87-3163$

\title{
DISCLAIMER
}

\begin{abstract}
This report was prepared $r$ an account of work sponcored by an asency of the United Sistee Government. Noither the Unitod States Gowernment nor any agency thereof, nor any of their employoes, makes any warranty, expreas of implised, of aseumes any legal liability or roeponaibility for the accuracy, completenese, or usofulness of any information, apparatus, product, of procese disclosed, or reprosents tiat its use would not infringe privately owned righte. Refer. ence herein to eny epecific commercial product. procese, or ecrvice by trade name, trademark. manufacturef, ot otherwise toes not nocesearily constitute or imply its endorsement, recommendation, or levoring by the United State Government of any agency thereof. The views and opinions of authors expreaced herein do not necescarily state of reflect those of the United State Government of ans agency thereof.
\end{abstract}




\author{
THREE-DIMENS IONAL, FREE-LAGRANGE HYDRODYNAMICS \\ HAROLD TREASE \\ LOS ALAMOS NATIONAL LABORATORY
}

\title{
1. INTRODUCTION :
}

The need to model $h$ igh speed fluid flow that involves gross material deformation and (local) high shear flow regions is becoming a requirement for computational flow morlels. These requirement.s dictate the use of robust, accurate numerical methods to model such fluid flow problems. Problems of this type have brought about the development of a new numerical technique called the FLM (Eree-Lagrange Method). The two-dimensional devilopment of this technique has been pioneered by crowley [1] and Fritts[2]. Thelr work has led to further cievelopment by clark[5] and Trease[4]. The first international conference on the FLM [5] was held In 1985 .

This paper describes the algorithms that make up a $3-D$ version of the FLN (the 2-D version is described in (4)). The basic method involves the explicit integration of the fluld flow equations over control volumes formed by Voronol cells. A Vuronol sell. is an arbitrary, convex polyhedron that has plane polygon faces. The "neareyt" nelghbors of any given mass polnt axe identified by noting the mass point that lies on the opposite side of a given Voionol face. The nearest nelghbors of a mass point are allowed to change in regponse to the (Lagrange) motion of the mags points. This implies that the mugh reorganizus itself as the mass points move with the Eluld motion.

As w1.l1 be described later the Voronol meshing techrique has several "nlce" features abcut it that impact the robustness and accuracy of the solution of the equations that are solved on this type of mesh. The following lo a zilch.iumary of some of thess ntce features:

1) A convex polyhedron giarantees that the control volumes are not distorted or reentrant. 
2) Each face of the polyhedra is described by arbitrary polygons zuch that each face intersects the line between two mass points as a perpendicular bisection plane. This implies that when forming the difference operators for any of the equations that (local'y) the mesh spacing is always "uniform".

3) The space defined by an arbitrary distribution of mass points plus any external boundaries is tessellated completely and uniquely by a set of Voronol polyhedra. Th1s tessella'ion is guaranteed to give. reciprocity of connertions.

4) The malntenance of the (Voronoi) connectivity matrix as the Lagrangian mass points foilcw the fluid motion is a matter of applying the Voronol "ruies" for (re) defining the nearest neighbors of a point. This leads to an erflcient and systematic algorithms for mairsaining the global connectivity matrix.

5) During the reconnection of thu mesh, where mass points change nearest neighbors, the integration control volumes for each mass point (1.e.. the Voronol polyhedra) do not change trejr topological form. This means that during the redefinition of the nearest neighbcrs of a mass point there is no need to "flux" megh quantities because of tho reconnections.

6) The distribution of mass points used to describe the goometry of a problem land used as the basis of the disc:etization method) can be locally refiniad to resolve fe. 'ures of $a$ problem. Iince a connectivity matrix la corstructed $f=0 m$ the mass point distriburion, and subsequertiy malritalned by a mesh optimization phase, we can add resolution as a problem evolves or we can remove rosilution if the need ariyes le.g., an example would be a tolme-step ciash raused by two mais polnts that come "too close").

In the reratrider of this paper I w11 the describe"the major stors " and solution algorithms that are used to solve an examole 3-dimingional ILuld flow problem. Also, I will present the results of this problein (NOTE: This problem 18 a standard strong shock, test problem, called 
the Noh problen [6]. This test problem represents just one $2 E+5=02-i$. verification prcblems in our overall quality/assurance procedure). The definition of the example problem to be used to describe the FIM is shown in Fig. 1. Here we have a infinite strength shock moving into an undisturbed fluid. The problem $1 \mathrm{~s}$ solved in plane geometry for the purposes of the this paper, but cylindrical and spherical versions are also used for code verification.

2. MESH GENERATION:

This step inciludes the inttial definition of a problem. The setup of a problem's geometry requires the following:

1) A distribution of mass points over the region of space.

2) The definition of any external boundaries.

3) The types of materlals in each material regioz along with Inttlal material densities, temperatures, etc.

An example distribution of points and the resulting voronoi mesh is shown in Fig. 2 (This is actually a self-generated distribution of points from a set of "seed" surface points and an automatic mesh refinement algorithm). Th1s flgure shows an arbitrary set of mass points and the corresponding Voronol polygons that ary constructed from the algorithm described by Trease [5].

The mesh that I will use in my example calculation(s) is shown in Figure $3 a-c$. Figure $3 a$ shows the geometry of $1-D$ version of the problem. Figure 3b shows the 2-D definition of the problem and figure 3 : shows the 3-0 setup for the same problem.

The main features to note about the tessellation method is that the entire space is mapped by a set of nor-ovarlapping convex polyhedia, where each mass polnt (located at the centor of the cells) ls separatod from its nearest nelghbors by perpendlcular bisecting, plane polygon E. $\mathrm{xceg}$. 
3. INTEGRATION METHOD:

- To solve our example hydrodynamics problem, we must solve the fluid flow equations by using the specified initial conditions and bourdar" conditions. The solution method that I choose to use involves the explicit time integration of the (discretized) fluid flow equations over a set of control volumes represented by the Voronoi control volumes.

The differential form of the fluid flow equations that represent the conservation of mass, momentum, and internal energy are given by:

1) Conservation of Mass (continuity equation);

$$
\frac{d \dot{\varphi} \rho}{\dot{k} t}=-\rho \vec{\nabla} \cdot \vec{u}
$$

2) Conservation of Momentum;

$$
\rho \frac{d \vec{u}}{d t}=-\vec{\nabla} p-\vec{D} \cdot \vec{\sim}
$$

3) Conservation of Internal energy:

$$
\begin{aligned}
& \rho \frac{d I}{c t}=-p \vec{\nabla} \cdot \vec{u} \cdot[\vec{\nabla} \cdot(\vec{r} \cdot \vec{u})-\vec{u} \cdot \vec{v} \cdot \vec{r}] \\
& \dot{S}=\text { pLUTO DENSTTR } \\
& \vec{u}=\vec{u}(x, y, \lambda)=\frac{d \vec{x}(x, y, \alpha)}{d t}=F \text { FU20 v } \\
& P=\text { MATERIAL PRESSUrE } \\
& \overrightarrow{\vec{Y}}=\text { (ARTZFZCZAL VISCOSITy) } \\
& \text { FLUId STRESS TENSOr } \\
& \vec{\nabla} \phi=\text { GHAOTENT O.PEAATPP } \\
& \vec{D} \cdot \vec{A}=\text { DEVERGENCA OPERATOR }
\end{aligned}
$$


These equations are spatially integrated over a control volume,

where we transform (on the right-hand side of the equations) the

volume integrals to surface integrals. These three equations now

take on the integral form:

1) Conservation of Mass (continuity equation);

$$
\begin{aligned}
& V \frac{d s}{d l}=-\bar{\rho} \int_{s} \hat{n} \cdot \vec{u}_{s} d S \\
& V=V O L U A E, d S=\text { SURFACE AREA FCEAENT } \\
& \hat{n}=\text { UNIT MURAL }
\end{aligned}
$$

2) Conservation of Momentum;

$$
\begin{aligned}
& m \frac{d \vec{l}}{d t}=-\int_{S}^{n} P_{S} d S-S_{S} \hat{n} \cdot \vec{r} d S \\
& m=S_{V} \rho d V=\text { mASS }
\end{aligned}
$$

3) Conservation of Internal energy;

$$
\begin{gathered}
m \frac{d z}{d t}=-\vec{p} \int_{S} \hat{n} \cdot \vec{u}_{S} d s-S_{S} \hat{n} \cdot(\overrightarrow{\vec{r}} \cdot \vec{u})_{s} d S^{\prime}+ \\
+\vec{u}_{S} \cdot S_{S} \hat{n} \cdot \vec{\gamma}_{S} d S
\end{gathered}
$$

These equations are now written in difference form as follows:

1) Conservation of Mass (continuity equation);

$$
\frac{\rho_{i}^{n+1}-\rho_{i}^{n}}{\Delta t}=\rho_{i} \sum_{j=1}^{J} \hat{n}_{i j} \cdot\left(\vec{u}_{s_{i j}}-u_{i}\right) \Delta S_{i j}
$$

$\dot{n}_{1 j}=$ UNIT NORMAL FROM POONT "j" To "j".

$\triangle S_{\text {Jj }}=$ SURFACE AREA ELCAE,T BETWEEN 2) Conservation of thomentum: POINTS $" \because "+j "$

$$
\begin{aligned}
m \cdot \frac{\vec{u}_{i}^{n+1}-u_{i}{ }^{\prime}}{\Delta t}= & -\sum_{j=i}^{J} \hat{n}_{i j}\left(p_{i j}-p_{i} \cdot \Delta S_{i j}+\right. \\
& -\sum_{j=1}^{j} \hat{n}_{i j}, \overrightarrow{\vec{r}}_{i j} \Delta S_{i j}
\end{aligned}
$$


3) Conservation of Internal energy:

$$
\begin{aligned}
& M_{1} \frac{I_{i=1}^{n+I_{i}}}{\Delta+}=-\vec{P}^{m+\delta} \sum_{j=1}^{D_{1}} \hat{n}_{1 j} \cdot\left(\vec{u}_{j}-\vec{u}_{i}\right) \Delta S_{j,}+ \\
& -\sum_{j=1}^{J} \hat{n}_{i j} \cdot(\overrightarrow{\vec{\tau}} \cdot \dot{\bar{u}})_{j,} \Delta S_{i j}+ \\
& +\vec{u}_{j}^{n+\frac{1}{2}} \cdot \sum_{j=1}^{J} \ddot{n}_{i j} \cdot \vec{\tau}_{i j} \Delta s_{i j}
\end{aligned}
$$

There are several things to note about these equations.

1) Local gradients are represented by the difference

of a given quantity between two nearest neighbors.

2) Since we are solving strong shockwave dominated problems

we must define an artificial viscosity [7]. This is

done in a tensor form by using the fluid stress tensor

as an artificial viscosity tensor, where the fluid viscosity

is replaced by an artificial viscosity [8]. This implies

that artificial fluid stress tensor in cartesian coordinates

has the form, where $\vec{T}$ is the unit tensor:

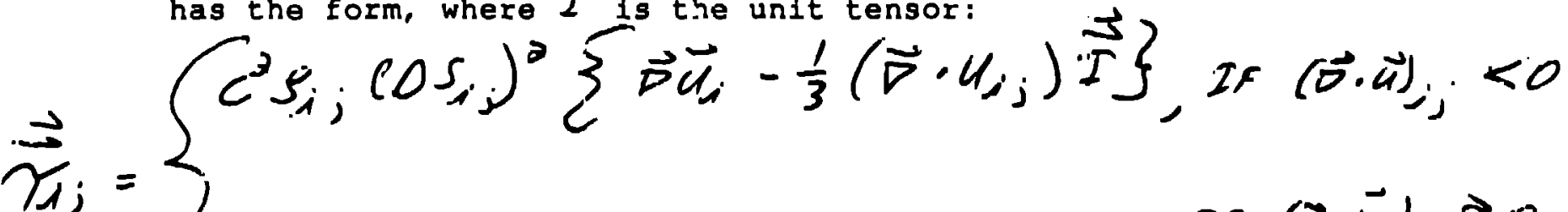

$$
\begin{aligned}
& \text { IF }(\partial \cdot \vec{u})_{i j} \geq 0
\end{aligned}
$$

3) Each pair of mass points are separated be a perpendicular

bisecting plane. This means that locally the mesh looks

like an equally spaced mesh. This fact enhances the (spatial)

accuracy of the differencing method, as can be shown by a

Taylor series expansion about one of the points.

For completeness, we note that in order for the solution method of the

three equations described above to be closed, we must have an equation-

of -state... whit ch relates the progsire af am material to the density and

Internal energy (or temperature) of a material. For our example problem

we use an ideal gas relation of the form: $P=(z-1) I S$

$\gamma=$ seton bias $L A W$

$-6-$

CONSTANT 
4. MESH OPTIMIZATION:

As our computational model follows the evolution of a fluid flow proolem, the discrete Lagrangian mass points move with with fluid motion. This process ordinarily results in a (hopelessly) tangled computational mesh. The power of the FLM is that the mesh can be reorganized by a reconnection algorithm to avoid this mesh tangling problem. The method that I use to reconnect the mesh is the same one used to tesselate the mesh in the generation phase. The "new" set of nearest nelghbors are (re)defined by the construction of the Voronol cell by using the "old" nearest neighbors and the nearest neighbors of the "old" nearest neighbors. This set of "possible" nearest neighbors is guaranteed to be complete due to the explicit (Courant time-step limited) nature of the time integration method (1.e., any single mass point cannot move out of its sphere of nearest neighbors in one computational cycle).

One of the most important features of using the Voronoi mesh to define the computational control volumes becomed apparent when a nearest neighbor reconnection occurs. The topology of the mesh doesn't change as a result of the reconnection. This implies that during this step the fluld volumes assoclated with the control volumes will not change. Thus, none of the physical quantitles assoclated with a given mass point (or voronol cell) need be fluxed between cells. Therefors, the numerical diffusion ordinarily associated with fluxing of conserved quantities is not a problem with a Voronot mesh based version of the FLM.

\section{RESULTS OF SOME CALCULATIONS:}

The example calculation that I will use to demonstrate the utility of the FLM is based on what we call the B1ll Noh problem [6]. This problem involves the generation of an infinite strength shock by a piston pushing lntu an undisturbed gas. The initial conditions and boundary conditions are shown in Fig. 1. Flguras $3 a-s$ shows the Voronol mesh for three variations of the Noh problell. Fig. 3a lo fust the mesh 
for a 1-D version of the problem. Fig. 3b is the mesh for a 2-D version - of the problem. For the 2-D run, I have intentionally introduced a mesh perturbation into the problem. The problem is still made of a uniform undizturbed gas, but the mesh is not uniform. This will test the code's ability to pass a $1-D$ shock wave through a non uniform mesh. The result should be a 1-D solution. Fig. 3c shows the final version of this problem where we run the same problem on a fully 3-dimensional mesh. The results for the $3-D$ case should be the same as for the 2-D case (i.e., the plane shock wave that is generated should remain $1-D$ even though the mesh has been perturbed. It should be noted that all of these problems were run on the same code. Only the point distributions were modified.

The results of the 1-D case are shown in Figures 4-7. The analytic solution to this problem gives the conditions of the shocked fluid as:
1) Shocked fluid density
$-4.0$
2) Shocked fluld pressure
$-1.33333333$
3) Shocked flutd (specific Internal) energy $=0.5$
4) Shocked fluid velocity
$-0.0$

The multidmensional results show a simllar state of the shocked fluid behind the shock front. Figure 8 shows the 3-D time-0 Voronol mesh. Figures 8-10 shnw the results of the 3-D calculation. Here the moving piston boundary moved from rlght to left. The effect of the mesh perturbation on the solution (indlcated by the stralghtness and magnitude of the contour lines) is minimal as the density contour plots show.

\section{CONCLUSIONS :}

In this paper I have given a brief outline of the Free-Lagrange Method as applied in three dimensions, where the computational control volumes are Voronol cells. I have described elsewhere how a voronol mesh can be generated from an arbitrary distribution of mass points [5] so I did not repeat that discussion. The results of a (simple) strong shock problem was presented showing the results in 1 and 3 -climensions. The 
results all look good, but this is a relatively easy problem to model.

The maln Idea that should be gleaned from this paper is that the FLM Is an extremely robust, accurate method for modelling fluid dynamics problems. Any geometry can be described, since the Voronoi mesh algorithm generates the connectivicy matrix needed to define the nearest neighbors of the points. Also, the method allows for adding or removing resolution based on the local mesh refinement conditions. Lastly, the method preserves it's accuracy, independent of the fluid distortions, due to the local reconnection algorithm.

\section{FUTURE WORK:}

The future of the FLM looks very bright for solving highly distorted hydrodynamic problems. As discussed at the last conference on the FLM [5] there are currently two distinct, but related, approaches to using the Voronoi mesh as the basis for a code. First, the Voronol mesh can be used to define both the nearest neighbor connectivity matrix and the topology of the computational control volumes. Second, the Voronoi mesh can be used to define the nearest neighbors but the control volume could be different from the Voronoi control volume. A good example of an alternative is to use the Median mesh to define the control volumes. A second alternative is to use the mesh that Pat Crowley describes in his paper for this same conterence publication.

As I have described in this paper a code based sompletely on the Voronoi mesh is possible and froduces good results for distorted flow problems. The mafor problem with the method is the computational speed of the resulting cude. The 3-D code, described in this paper, consumes vast amounts of computer time on our largest computers here at Los Alamos. Therefore, the 3-D code team has embarked on a project whereby we will rewrite the code to Improve the speed of the aljorithms, but still retain the Free-Lagrange splrt.

The rewriting of the codo is in progress as I write this paper. To Improve the speed of the code we have made the following changes:

1) The basic topological element of the mesh is now a tetrahedron 
rather than a polyhedron. Each tetrahedron represents one element of the Delaunay mesh (Note: The Delaunay mesh is the dual to the Voronol mesh.)

2) The nearest neighbor generation and maintenance algorithms work with tetrahedrons. The resulting nearest neighbors are still the Voronoi nearest neighbors.

3) The integration of the hydrodynamic equations is perf rmed with a predictor-corrector time stepping algorithm and over control volumes defined by the Median mesh (but using the Voronoi nearest neighbor connectivityl.

The prognosis for the new algorithm is that it is significantly faster than the old one, but the results are comparable. Future publications will detall the results of detalled comparison between the: Median and Voronoi algorithms.

\section{REFERENCES :}

[1] W. P. Crowley, "FLAG: A Free-Lagrange Method for Numerically Simulating Hydrodynamic Flows in Two Dimensions", Lecture Notes in Physics 8, 37-13, Springer (1970).

[2] M. J. Fritts, J. P. Boris, "The Lagrangtan Solution of Transient Problems in Hydrodynamics using a Triangular Mesh", J. Comp. Phys. 31, 173-?15 (1979).

[3] "The Free-Lagrange Method", Lecture Notes in Physics 238, 281-294, Springer (1985).

[4] H. E. Trease, "A Two-Dimensional Free Lagrangian Hydrodynamics Model", Ph.D. Thesis, University of Illinols, Urbana-Champaign (1981).

ii] "Ihe Free-Lagrange Methcd", Lecture Notes in Physics 238, Springer (1985).

[6] W. F. Noh, "Artiflcial Viscosity for Strong Shocks", Lawrence Livermore Naclonal Laboratory, Livermore CA., UCR.L-53669.

[7] J. Von Neumann, R. D. Richtmyer, "A Metiod for the Numerical Calculation of Hydrodynamic Shocks", J. Appl. Phys., 21, 232 (1957).

[8] W. M. Tscharnuter and K. H. Winkler, "A Method for Computing Selfgravitation Gas Flow with Radjation", MPI/PAE-Astro 163. 


\section{FIGURE CAPTIONS :}

Figure 1: A description of the Noh problem in plane geometry.

Figure 2: Four views associated with the generation of a Voronol mesh.

- Upper left is the inital point distribution.

- Upper right is the Voronoi mesh.

- Lower left is a rotated view of the Voronol mesh.

- Lower right is the Delaunay mesh (dual of the Voronol).

Figure 3: The Voronol meshes showing the 1-D, 2-D and 3-D setups.

Figures 4-7: The resujts for the 1-D Noh problem.

Figure 4: Density versus distance for the 1-D Noh problem at time-0.6.

Figure 5: Energy versus distance for the 1-D Nch problem at $t 1 m a-0.6$.

Figure 6: Pressure versus distance for the 1-D Noh problem at time -0.6 .

Figure 7: Velocity versus distance for the 1-D Noh problem at time-0.6.

Figures 8-10: The results for the 3-D Noh problem.

Figure 8: The time-0.0 setup for the 3-D Noh problem with a mesh perturbation imbedded in the problem.

Figure 9: The time-0.38 results of the ?-D Nuh problem that shows the position of the shock from in relation to the mesh.

Figure 10: The time-0.38 results of the 3-D Noh problem that shows the position of the shock from in relation to the mesh. The positior of the shock front is indicated by the leading edge (moving right to left) straight contours. 


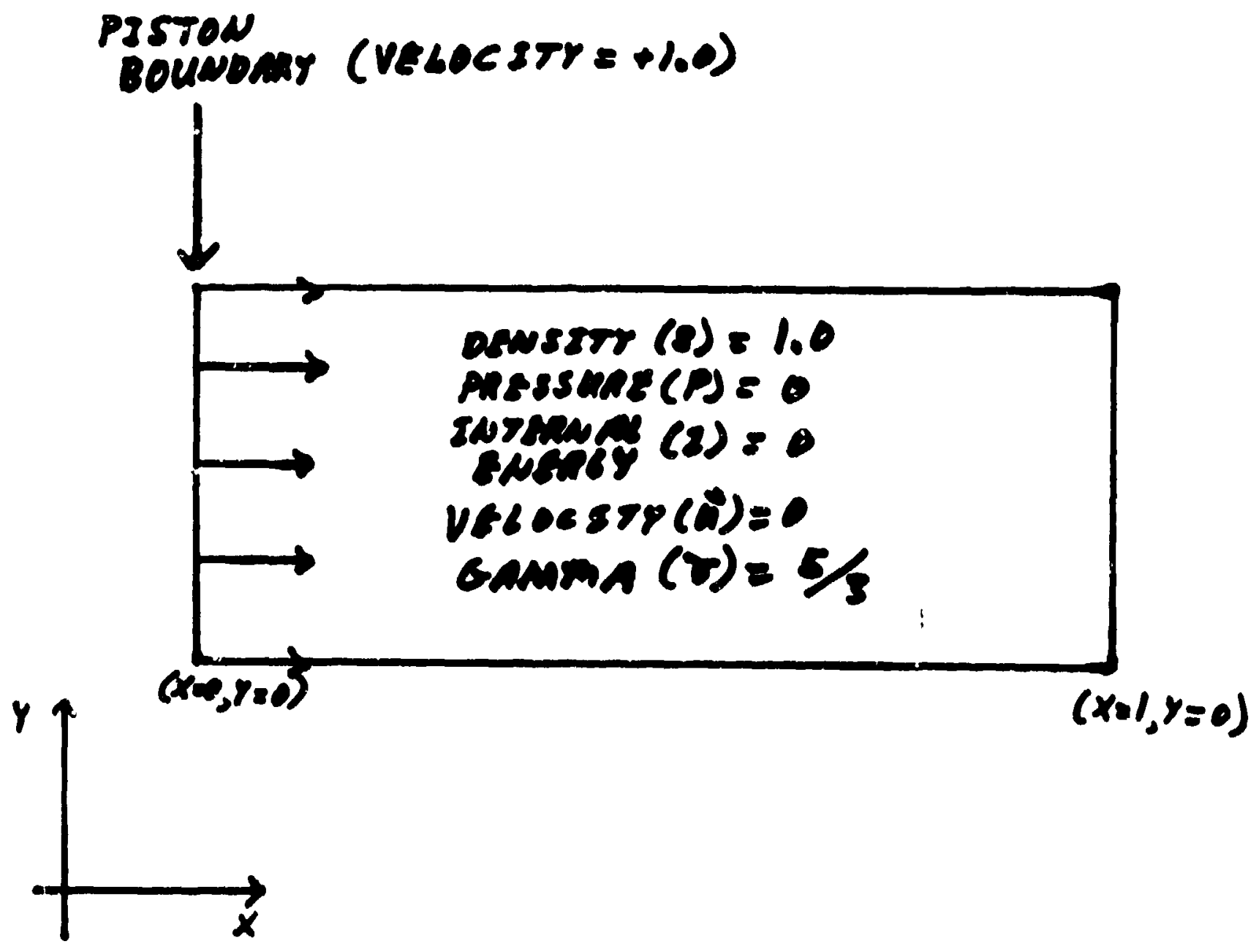

Flgure 1: A description of the Noh problem in plane geomet"y. 


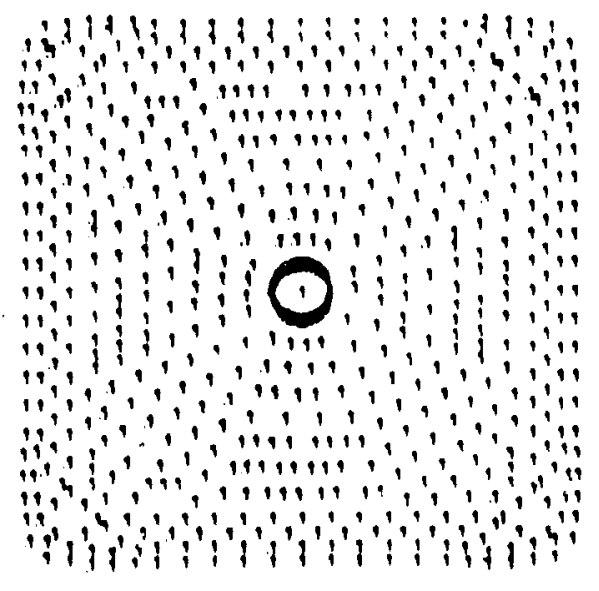

POINT DISTRIBUTION

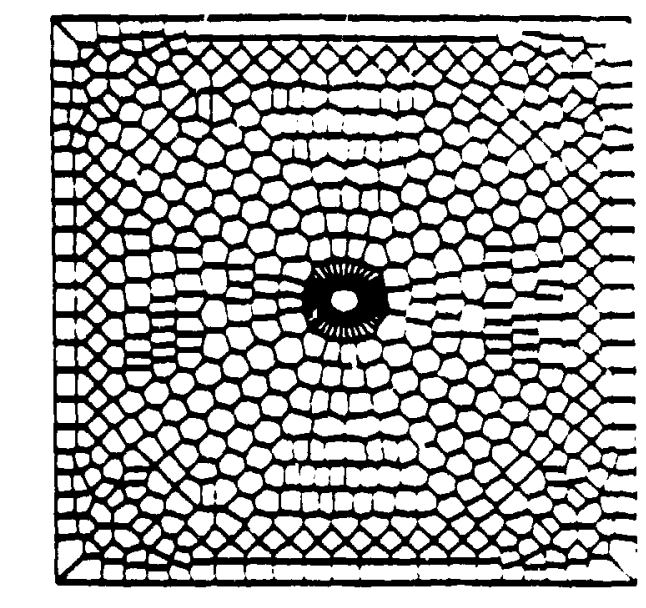

VORONOI MESH

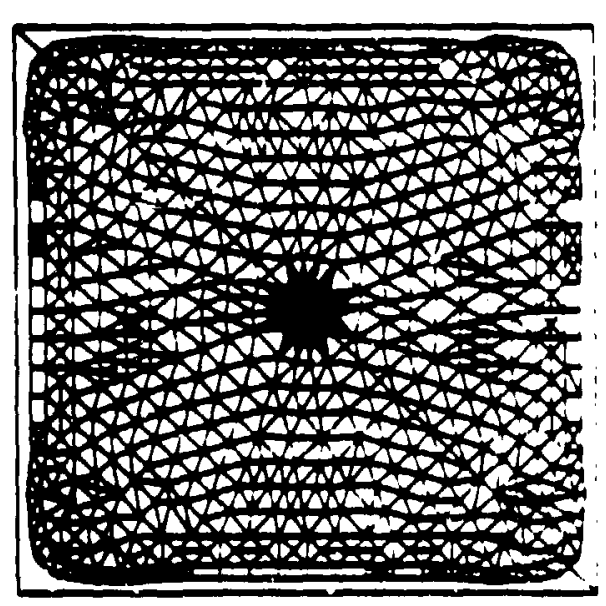

DELAUNAY MESH

(1)

(ROTATED) VORONOI MESH

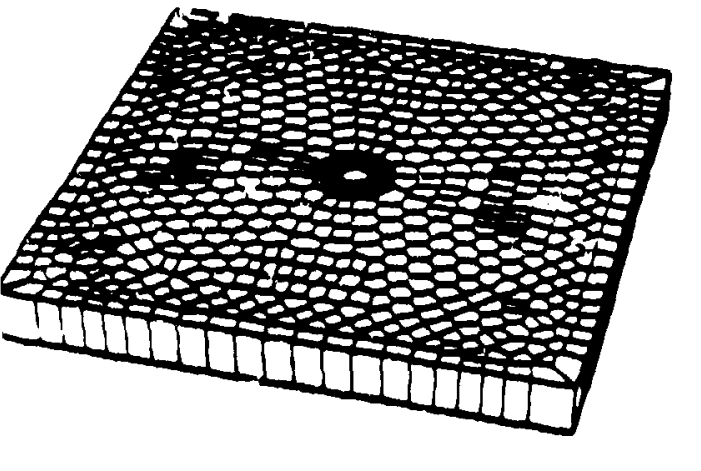

Figure 2: Four views assoclated with the generation of a voronol mesh.

- Upper left is the inital point distribution.

- Upper right is the Voronoi mesh.

- Lower left is a rotated view of the voronol mesh.

- Lower right is the Delaunay mesh (dual of the Voronoi). 


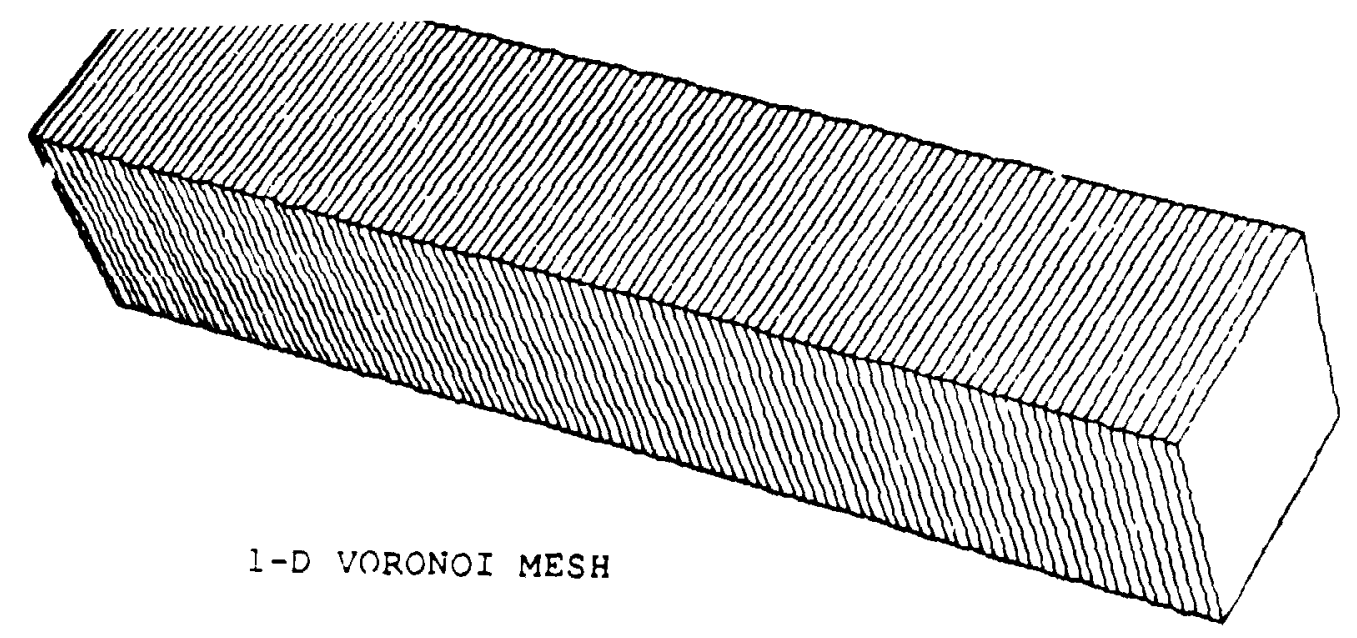

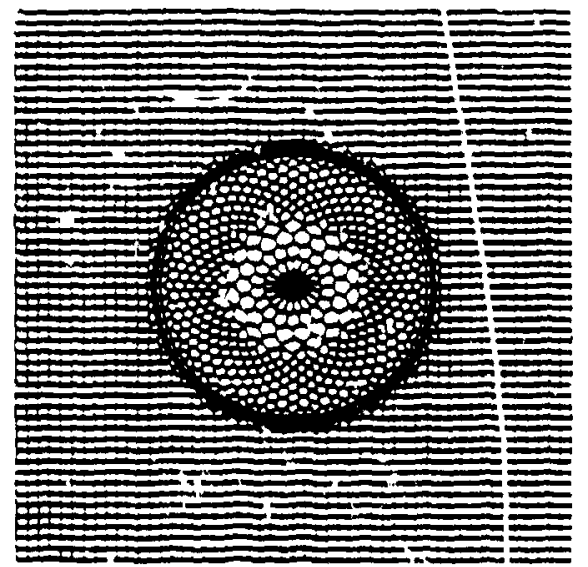

2-D VORONOI MESH

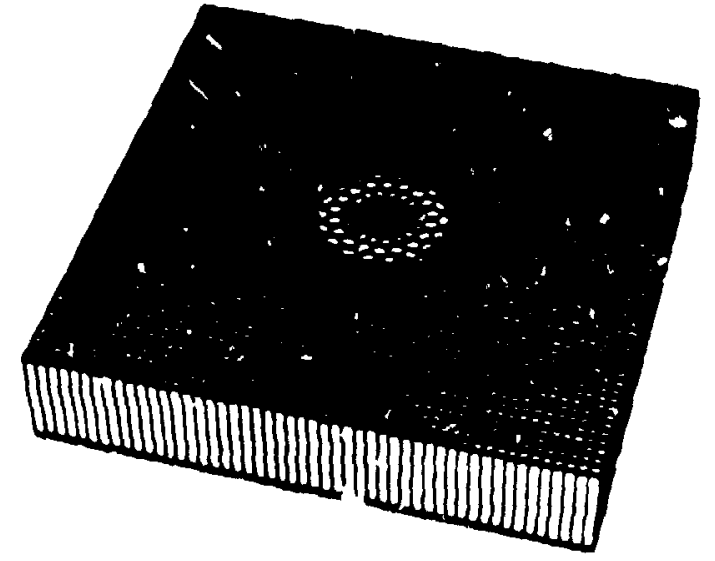

(ROTATED) 2-D PROBLEHA

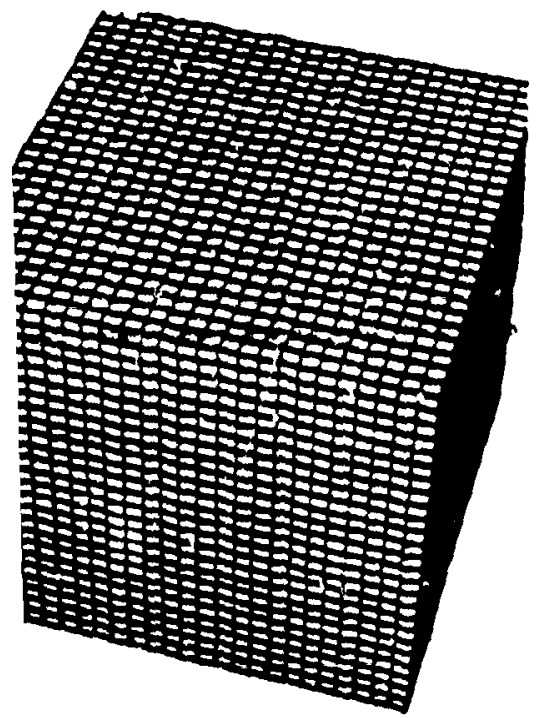

ORONOI MESH

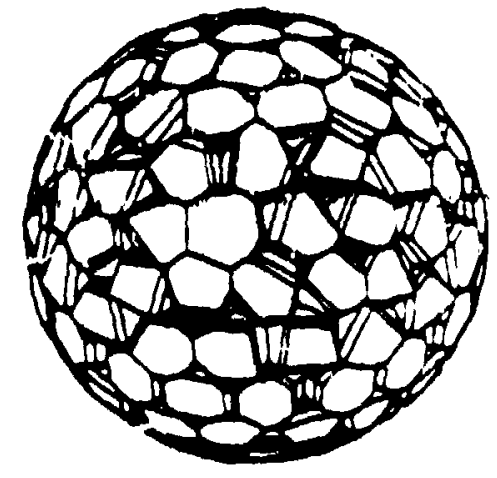

VORONOI MESH FOR IMBEDDED BALC

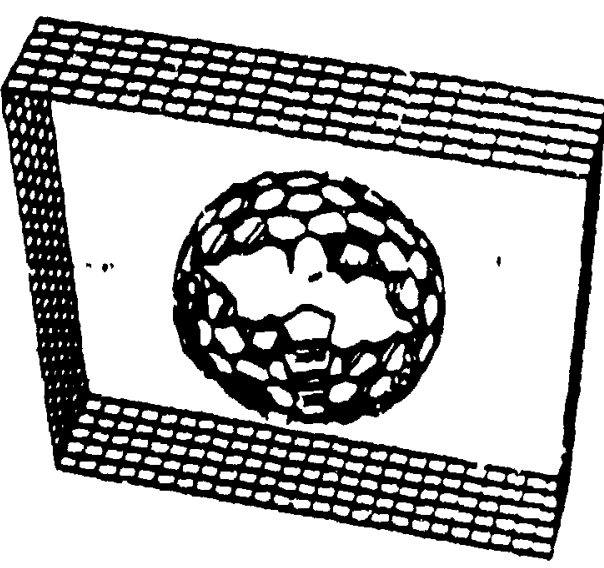

CLIPPED VORONOI MESH

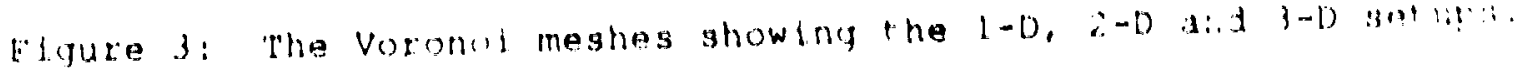




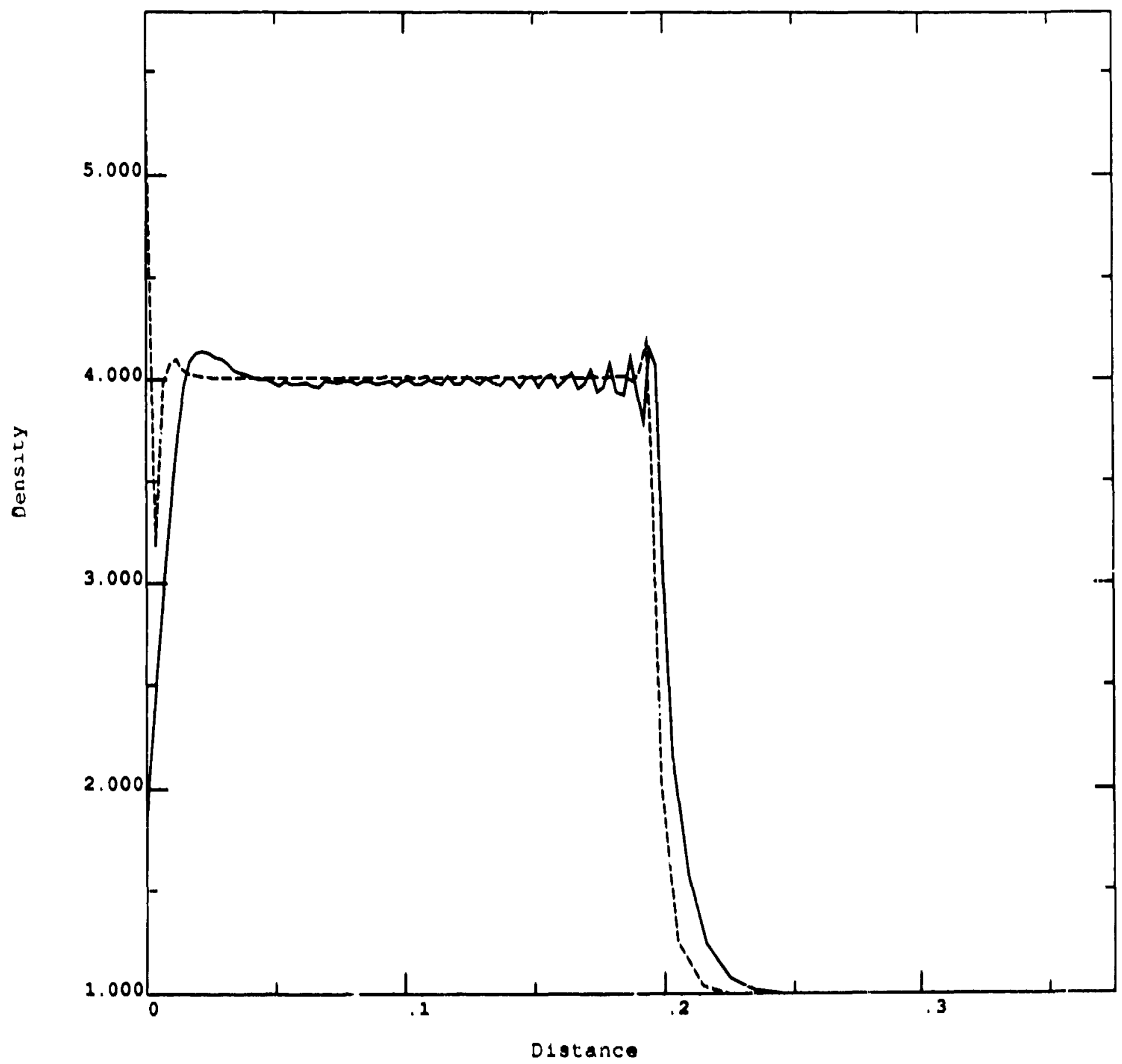

Figure 4: Density versus distance Lor the 1-D Nuh problem at time-0.6. 


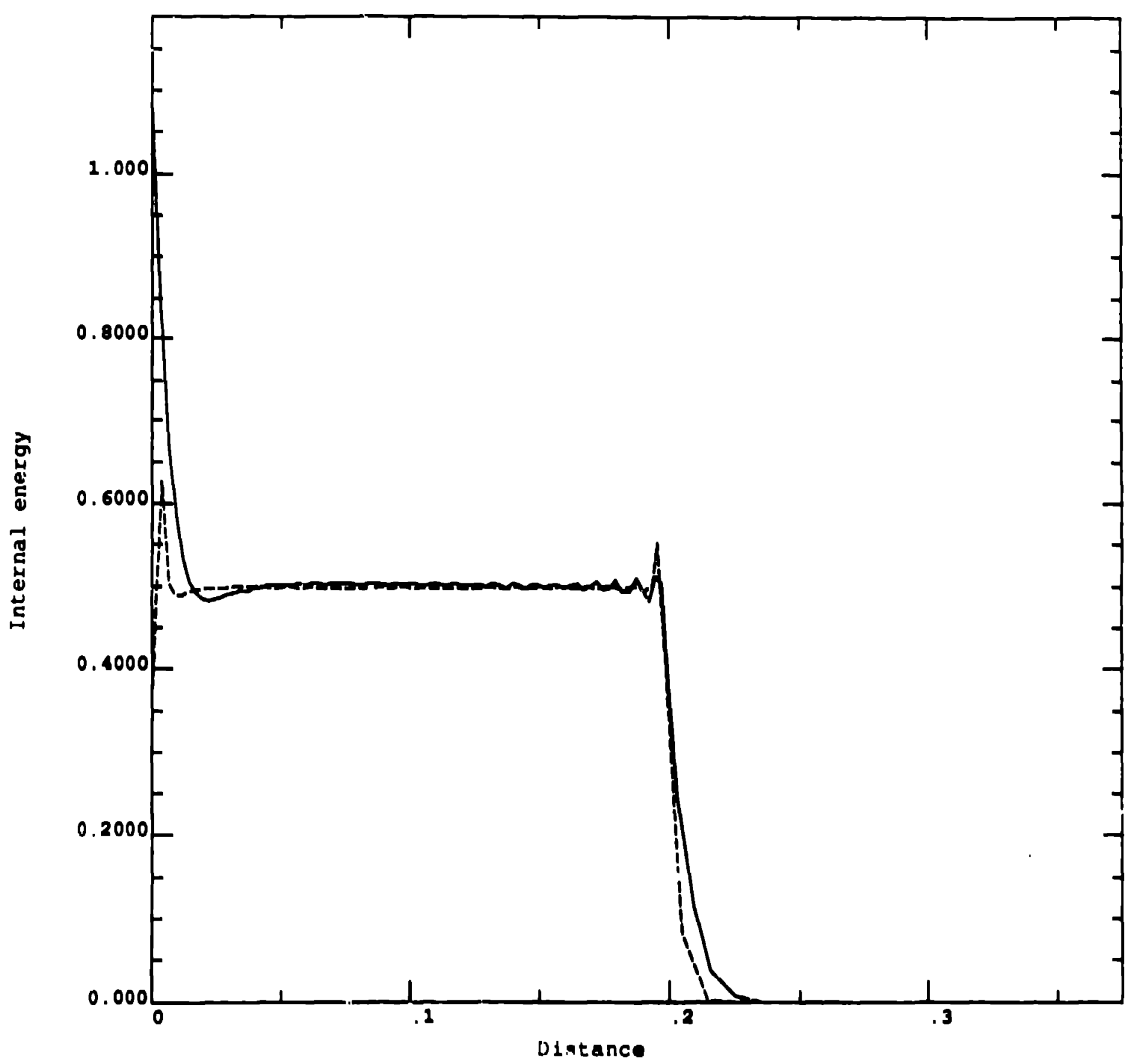

Flgure 5: Energv varaus distanch cor the 1-D Nah priotrom at time $\cdot 0.6$. 


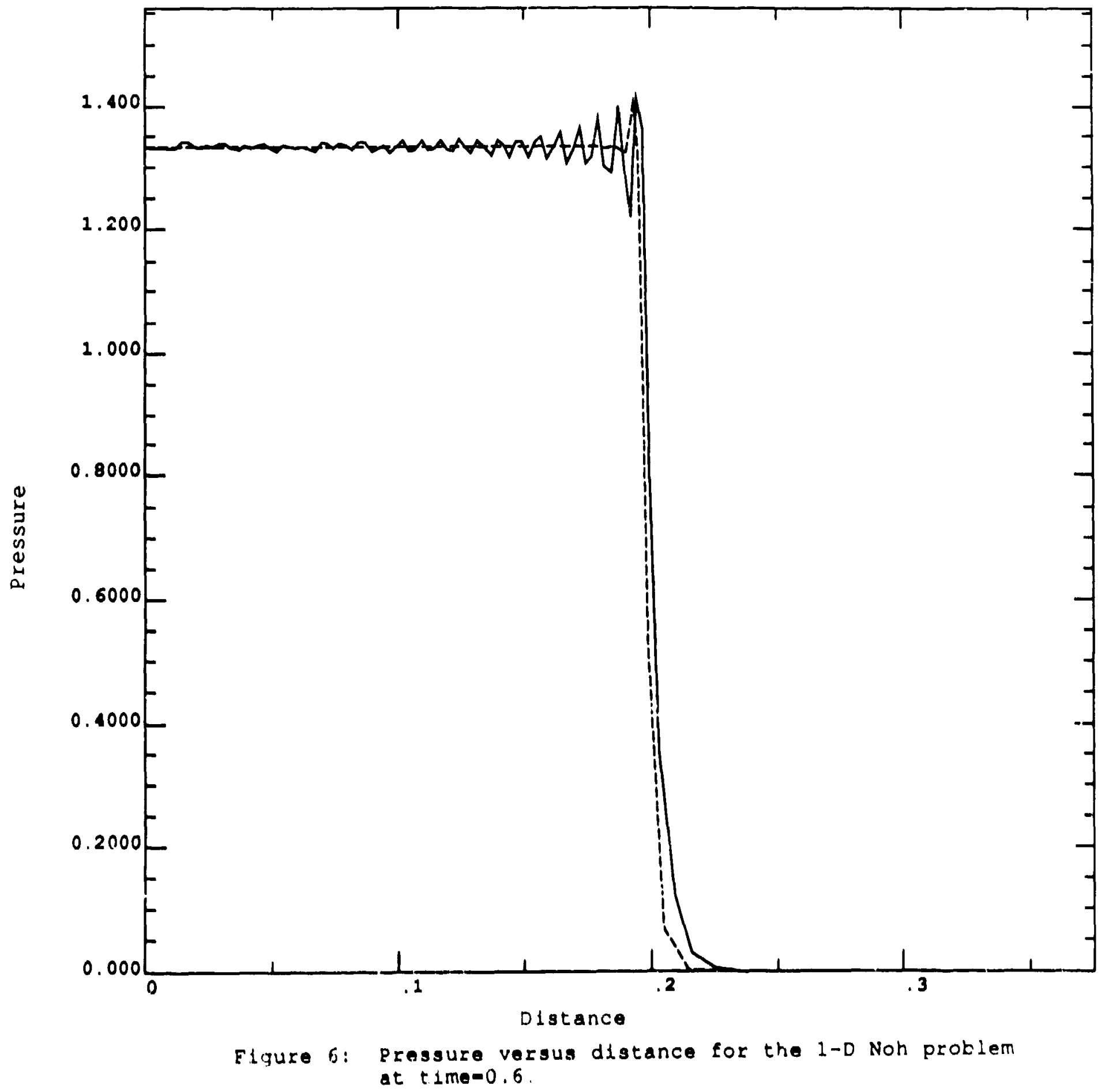




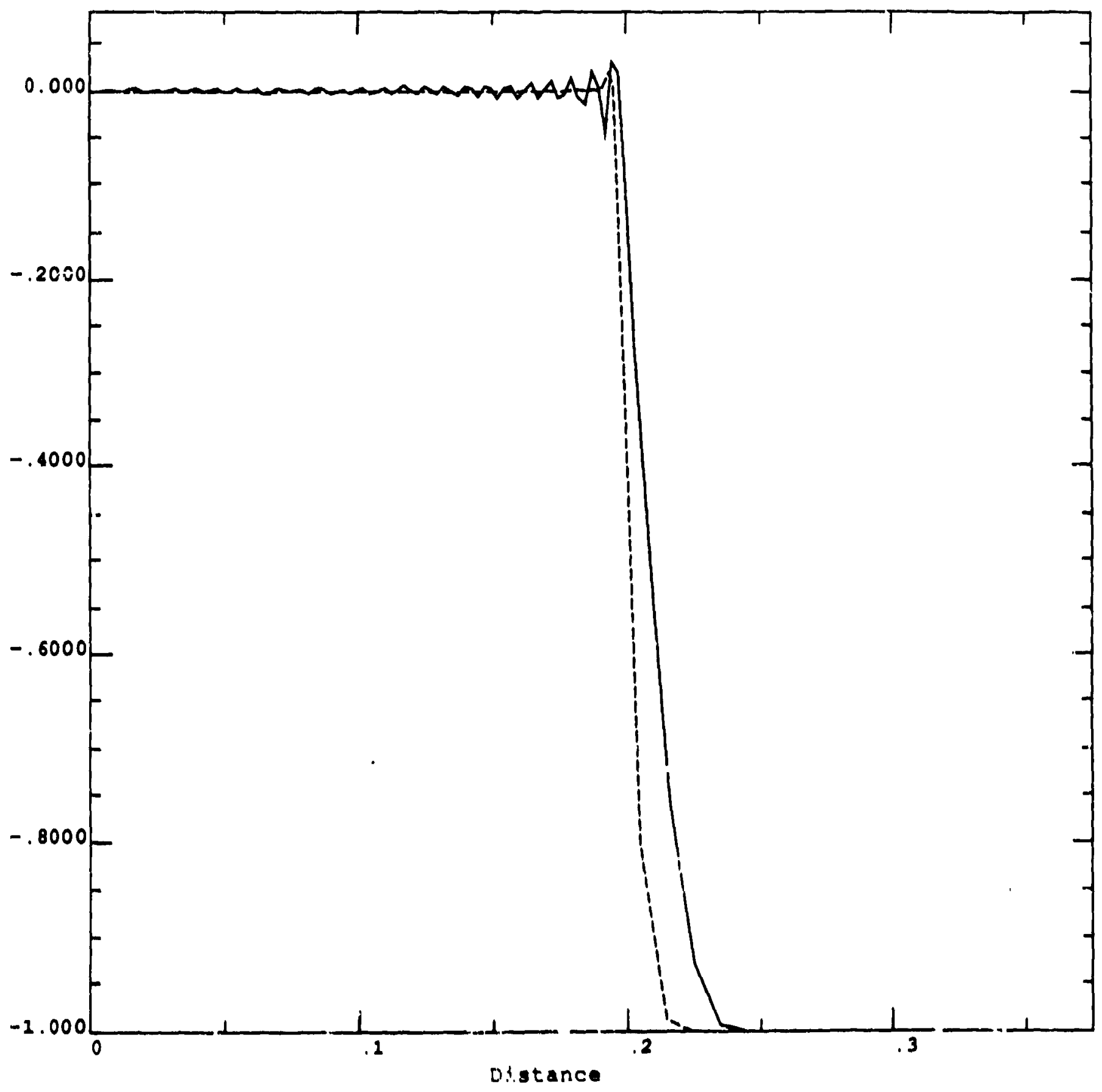

Figure 7: Velocicy versus distance for the 1..D Noh problem at time $=0.6$. 